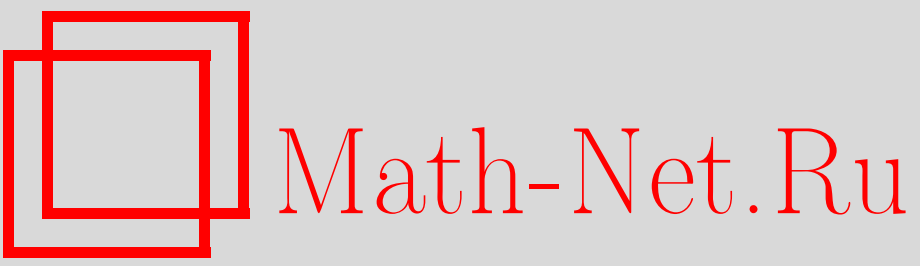

Ю. В. Еремейченкова, Е. В. Зароченцев, Е. П. Троицкая, Новый подход к расчету энергетической структуры кристаллов, основанный на кластерном разложении, ТМФ, 1996, том 106, номер 3, 498-512

DOI: https://doi.org/10.4213/tmf1132

Использование Общероссийского математического портала Math-Net.Ru подразумевает, что вы прочитали и согласны с пользовательским соглашением

http://www.mathnet.ru/rus/agreement

Параметры загрузки:

IP : 54.198 .64 .247

26 апреля 2023 г., 17:45:08 
ТЕОРЕТИЧЕСКАЯ

И МАТЕМАТИЧЕСКАЯ

ФИЗИКА

Том 106, № 3

март, 1996

Ю.В. Еремейченкова, Е.В. Зароченцев, Е. П. Троицкая

\section{НОВЫЙ ПОДХОД К РАСЧЕТУ ЭНЕРГЕТИЧЕСКОЙ СТРУКТУРЫ КРИСТАЛЛОВ, ОСНОВАННЫЙ НА КЛАСТЕРНОМ РАЗЛОЖЕНИИ}

Развивается новый подход к расчету энергетических спектров сильносжатых или несовершенных кристаллов, в которых перекрытие между локализованными орбиталями (LO) разных атомов велико. Подход основан на точной ортогонализации по Левдину всех LO кристалла, дополненной кластерным разложением (CE). В каждом $n$-м порядке СЕ учитываются интегралы перекрытия $(S)$ локализованных орбиталей атомов или остовов, принадлежащих одному и тому же $n$-частичному кластеру, а всевозможные $n$-частичные кластеры перебирают весь кристалл. Кластерное разложение быстро (как $S^{n}$ ) сходится по $n$. Подробно рассмотрено приближение двухчастичных кластеров.

\section{1. ВВЕДЕНИЕ}

При сильных сжатиях кристалла возникает необходимость учета перестройки по мере увеличения электронной плотности атомов в кристалле по сравнению с изолированными. Однако сушествуюшие методы исследования зонных структур (см., например, [1]) основаны на приближении "жесткого" остова. Это приближение дает хорошие результаты при самосогласованных расчетах зонной структуры в случае небольших сжатий, когда система уровней остова сушественно не меняется. В настояшее время практически отсутствуют обоснованные неэмпирические методы расчета энергетической структуры твердых тел, эффективные при экстремальных условиях.

Формально задача учета перекрытия волновых функций решается с помошью точной ортогонализации их по Левдину. В этом (и в использовании кластерного разложения (cluster expansion - CE)) заключается предложенная в данной работе модификация методов локализованных орбиталей (CELO) [7] и ортогонализованных плоских волн (CEOPW). В методе CELO предлагается использовать в качестве базиса ортогонализованные друг к другу по Левдину [6] атомные орбитали. В методе CEOPW в качестве базиса используются плоские волны, ортогонализованные к блоховским функциям занятых (или остовных) состояний кристалла, построенным в базисе метода CELO. Метод CELO эффективен для расчетов в условиях высоких давлений энергетических спектров кристаллов с сильной связью, а также остовных состояний металлов и полупроводников, а метод CEOPW - для расчета валентных зон последних, а также зон проводимости. 
В отличие от молекул для систем с большим числом атомов ортогонализация по Левдину является технически неосушествимой. Поэтому предлагается применение кластерного разложения, которое снимает эту трудность. Развитое в данной работе СЕ аналогично предложенному Абаренковым и Антоновой [8-10] для электронной плотности кристалла. Кластерное разложение учитывает перекрытие LO различных групп атомов (названных кластерами): двухчастичных, трехчастичных и т.д. Кластеры в совокупности перебирают весь кристалл. В низших порядках СЕ задача ортогонализации по Левдину LO всего кристалла сводится к аналогичным задачам для орбиталей кластеров с конечным (как правило небольшим) числом частиц. Подробно рассмотрено приближение двухчастичных кластеров.

\section{2. МЕТОД СЕLО}

Во всех расчетах методом LCAO (см. [1] и приведенные там ссылки) пренебрегали поправками, связанными со степенями интегралов перекрытия базисных LO из-за малости последних в несжатом кристалле. Однако даже в этом случае интегралы перекрытия между LO остовных и валентных электронов, являясь малыми, входят в уравнения LCAO умноженными на число ближайших соседей $z \sim 10$ и на энергию $E_{c}$ остовных электронов, большую по сравнению с зонной энергией $[2,3]$.

Поэтому в сжатых кристаллах интегралы перекрытия орбиталей (всех электронов, включая и остовные) дают заметный вклад в зонную энергию [4]. При больших сжатиях ясна необходимость учета ортогонализационных поправок более высоких, чем первого [4], порядков. В связи с этим прежде всего необходимо построить блоховскую функцию электрона кристалла, точно учитывая перекрытие между всеми локализованными орбиталями.

В качестве базиса для расчетов методом CELO будем использовать ортонормированньй набор LO $\chi_{1 s}$, построенных из неортогональных атомных орбиталей $\varphi_{\mathbf{l}^{\prime} s^{\prime}}$ с помощью ортогонализации по Левдину [10]

$$
\chi_{\mathbf{l} s}=\sum_{\mathbf{1}^{\prime}} \sum_{s^{\prime}} \varphi_{\mathbf{1}^{\prime} s^{\prime}}(\mathbf{r})\left(T^{-1 / 2}\right)_{\mathbf{I}^{\prime} s^{\prime} ; \mathbf{l} s} .
$$

Здесь $\mathbf{l}, \mathbf{l}^{\prime}$ пробегают все узлы решетки, $N$ - их число (рассматривается кристалл с одним атомом на ячейку), $s \equiv(n, l, m)$ нумерует занятые состояния атома. Будем считать, что каждое одночастичное состояние (их всего $Z / 2, Z$ - заряд ядра) занято двумя электронами с противоположно направленными спинами. Разделение на остовные и валентные электроны не делается.

Предполагается, что орбитали одного атома удовлетворяют стандартному уравнению Шредингера. В разложении (1) $T$ - так называемая метрическая матрица размера $L \times L(L=N \cdot Z / 2)$ с интегралами перекрытия между соответствующими орбиталями в качестве элементов

$$
T_{\mathbf{l}^{\prime} s^{\prime} ; \mathbf{l} s}=\int \varphi_{\mathbf{1}^{\prime} s^{\prime}}^{*}(\mathbf{r}) \varphi_{\mathbf{l} s}(\mathbf{r}) d^{3} r, \quad \mathbf{1}, \mathbf{l}^{\prime}=1, \ldots, N, \quad s, s^{\prime}=1, \ldots, Z / 2 .
$$

Матрица $T$ эрмитова. В (1) использована матрица $T^{-1 / 2}$ такая, что $T^{1 / 2} \cdot T^{-1 / 2}=I$, $T^{1 / 2} \cdot T^{1 / 2}=T$ и $T^{1 / 2} \rightarrow I$ при $T \rightarrow I$. 
Следующим шагом является построение по набору LO (1) блоховских функций

$$
\psi_{\mathbf{k} s}(\mathbf{r})=N^{-1 / 2} \sum_{\mathbf{l}} \exp (i \mathbf{k} \mathbf{l}) \chi_{\mathbf{l} s}(\mathbf{r})
$$

и пробных функций метода CELO

$$
\Psi_{\mathbf{k} v}(\mathbf{r})=\sum_{s} \psi_{\mathbf{k} s}(\mathbf{r}) C_{s v}
$$

где $C_{s v}$ - искомые вариационные параметры, индекс $v$ нумерует заполненные зоны. Чтобы пробные функции $\Psi_{\mathbf{k} v}(\mathbf{r})$ были ортонормированными, необходимо и достаточно, чтобы матрица коэффициентов $C$ была унитарной, $\sum_{s} C_{s v^{\prime}}^{*} C_{s v}=\delta_{v^{\prime} v}$. Это автоматически обеспечивается правильной симметрией функций $\Psi_{\mathbf{k} v}$ относительно точечной группы кристалла.

Варьируя выражение $E_{\mathbf{k} v}=\left\langle\Psi_{\mathbf{k} v}|H| \Psi_{\mathbf{k} v}\right\rangle /\left\langle\Psi_{\mathbf{k} v} \mid \Psi_{\mathbf{k} v}\right\rangle$ для зонной энергии по $C_{s v}^{*}$, приходим к следующей системе уравнений для параметров $C_{s v}$ :

$$
\sum_{s^{\prime}}\left\{\left\langle\psi_{\mathbf{k} s}|H| \psi_{\mathbf{k} s^{\prime}}\right\rangle-E_{\mathbf{k} v} \delta_{s s^{\prime}}\right\} C_{s^{\prime} v}=0
$$

Здесь $H=T+V(\mathbf{r})$ - гамильтониан электрона в кристалле, в котором $V(\mathbf{r})$ включает в себя взаимодействие со всеми ядрами (а не с остовами) и самосогласованное взаимодействие с остальными электронами кристалла в одноэлектронном приближении. Потенциал $V(\mathbf{r})$ обычно представляют в виде суперпозиции $V(\mathbf{r})=\sum_{\mathbf{l}} v_{\mathbf{l}}$, где $v_{\mathbf{l}} \equiv v(|\mathbf{r}-\mathbf{l}|)$ - самосогласованный потенциал, центрированный на узле $\mathbf{1}$. Энергия $E_{\mathbf{k} v}$ зонного электрона находится при решении секулярного уравнения системы (4) порядка $Z / 2$.

\section{3. КЛАСТЕРНОЕ РАЗЛОЖЕНИЕ ДЛЯ МЕТОДА СЕLО}

Для решения системы (4) необходимо рассчитать матричные элементы $\left\langle\psi_{\mathbf{k} s}|H| \psi_{\mathbf{k} s^{\prime}}\right\rangle$. Для конкретных расчетов с точной ортогонализацией по Левдину разовьем кластерное разложение, аналогичное предложенному Абаренковым-Антоновой в [8-10].

Сначала преобразуем функцию $\psi_{\mathbf{k} s}$ к виду, удобному для введения СЕ. Подставим $(1)$ в $(3)$ и выделим из $\psi_{\mathbf{k} s}$ ту ее часть $\psi_{\mathbf{k} s}^{(0)}(\mathbf{r})$, которая получилась бы в пренебрежении всеми интегралами перекрытия:

$$
\begin{gathered}
\psi_{\mathbf{k} s}(\mathbf{r})=\psi_{\mathbf{k} s}^{(0)}(\mathbf{r})+\Delta \psi_{\mathbf{k} s}(\mathbf{r}), \quad \psi_{\mathbf{k} s}^{(0)}(\mathbf{r})=N^{-1 / 2} \sum_{\mathbf{l}} \exp (i \mathbf{k} \mathbf{l}) \varphi_{\mathbf{l} s}(\mathbf{r}), \\
\Delta \psi_{\mathbf{k} s}(\mathbf{r})=-N^{-1 / 2} \sum_{\mathbf{l}} \exp (i \mathbf{k} \mathbf{l}) \sum_{\mathbf{l}^{\prime} s^{\prime}} \varphi_{\mathbf{l}^{\prime} s^{\prime}}(\mathbf{r}) \mathscr{P}_{\mathbf{l}^{\prime} s^{\prime} ; \mathbf{l} s}, \quad \mathscr{P}=I-T^{-1 / 2} .
\end{gathered}
$$

В [8-10] была выведена основная формула СЕ для произвольной матрицы $A_{t}$, сопоставленной кристаллу,

$$
A_{t}=\sum_{n=1}^{N} \sum_{[n] \subseteq[N]} Q_{t}[n], \quad Q_{t}[n]=\sum_{m=1}^{n} \sum_{[m] \subseteq[n]}(-1)^{m+n} A_{t}[m] .
$$


Здесь и далее $[\eta] \equiv\left[\mathbf{l}_{1} \ldots \mathbf{l}_{\eta}\right]-\eta$-частичный кластер, $[N] \equiv[1 \ldots N]$ - весь кристалл. Вторые суммирования в $(7)$ ведутся по всевозможным кластерам $[n] \subseteq[N]$ (или $[m] \subseteq$ $[n])$, перебирающим больший кластер. Порядок следования атомов в кластере не важен. $\mathrm{B}$ дальнейшем нам понадобятся матришы вида $A_{t}=I-T^{-t}$, причем $A_{1 / 2}=\mathscr{P}(6)$, а $A_{1}=P(16)$ (см. ниже),

$$
A_{t}[m]=\left\{\begin{array}{l}
\mathscr{P}[m], \quad t=1 / 2 \\
P[m], \quad t=1
\end{array}=\left(I-(T[m])^{-t}\right), \quad A_{t}[N] \equiv A_{t} .\right.
$$

Хотя в [10] была выведена основная формула кластерного разложения для произвольной матрицы $A$, теоремы, даюшие возможность практического использования этого разложения, доказаны там лишь для частного случая $t=1$, необходимого для вычисления матрицы плотности. Формулы (6) для блоховских функций, а значит, и выражение для энергии зонного электрона содержат матрицу $\mathscr{P}$. Следовательно, справедливость теорем, доказанных для матрицы $P$ в $[10]$, придется заново доказывать для $\mathscr{P}$.

Все матришы в $(7)$ - размера $L \times L$. Через $T[m]$ обозначена кластерная метрическая матрица. По определению [8-10] она имеет вид

$$
T[m]_{\mathbf{1} s ; \mathbf{1}^{\prime} s^{\prime}}= \begin{cases}T_{\mathbf{l} s ; \mathbf{1}^{\prime} s^{\prime}}, & \mathbf{1}, \mathbf{1}^{\prime} \in[m], \\ \delta_{\mathbf{1 \mathbf { l } ^ { \prime }}} \delta_{s s^{\prime}} & \text { в противном случае. }\end{cases}
$$

Для последуюшего рассмотрения полезно представить $T[m]$ в виде

$$
T[m]=I+S[m],
$$

где $S[m]$ - кластерная матрица интегралов перекрытия с элементами

$$
S_{\mathbf{l} ; ; \mathbf{1}^{\prime} s^{\prime}}=T_{\mathbf{l} s ; \mathbf{1}^{\prime} s^{\prime}}-\delta_{\mathbf{1 1 ^ { \prime }}} \delta_{s s^{\prime}} .
$$

Все матрицы с элементами вида $A_{1 s ; \mathbf{1}^{\prime} s^{\prime}}$ удобно представлять в блочном виде [10]

$$
A=\left\|\begin{array}{ccc}
A_{\mathbf{l}_{1} ; \mathbf{l}_{1}} & \ldots & A_{\mathbf{l}_{1} ; \mathbf{l}_{N}} \\
\ldots & \ldots & \ldots \\
A_{\mathbf{l}_{N} ; \mathbf{l}_{1}} & \ldots & A_{\mathbf{l}_{N} ; \mathbf{l}_{N}}
\end{array}\right\|,
$$

где каждьй блок $A_{\mathbf{1} ; \mathbf{1}^{\prime}}$ - квадратная матрица порядка $(Z / 2)$ с элементами $A_{\mathbf{l} s ; \mathbf{1}^{\prime} s^{\prime}} ; \mathbf{1}$ и $\mathbf{l}^{\prime}$ фиксированы. В дальнейшем, следуя [8-10], будем называть блоки с $1, \mathbf{l}^{\prime} \in[m]$ блоками типа I относительно кластера $[m]$, а блоки с $\mathbf{l} \mathbf{l}^{\prime} \notin[m]$ - блоками типа II относительно данного кластера.

Можно показать (см. приложение 1$)$, что матрица $(T[m])^{-1 / 2}$ обладает следуюшими свойствами:

1) для недиагональных блоков типа II $(T[m])_{1: 1^{\prime}}^{-1 / 2}=0$, а для диагональных блоков типа II $(T[m])_{\mathbf{1} ; \mathbf{1}^{\prime}}^{-1 / 2}=I$; здесь 0 и $I-$ нулевая и единичная матришы размера $Z / 2 \times Z / 2$;

2 ) для извлечения квадратного корня из матришы $T[m]$ достаточно решить характеристическое уравнение $\operatorname{det}\|\mu I-T[m]\|$ меньшего порядка (равного $m \cdot(Z / 2)$ вместо $N \cdot(Z / 2)$, как для полной метрической матришы). 
Матрица $Q_{1 / 2}[n]$ (7) обладает следуюшими свойствами:

1) все блоки типа II матрицы $Q_{1 / 2}[n]$ равны нулю. Это свойство следует из свойства 1 матрицы $(T[m])^{-1 / 2}$ и определения (7) матрицы $Q_{1 / 2}[n]$. Благодаря этому суммирование по всем узлам кристалла $\mathbf{l}$ и $\mathbf{l}^{\prime}$ в (6) сводится к суммированию по $\mathbf{l}, \mathbf{l}^{\prime} \in[n]$ в каждом из слагаемых в (9) (см. ниже), что значительно упрошает расчеты;

2 ) матрица $Q_{1 / 2}[n]$ равна нулю, если орбитали хотя бы одного из атомов кластера $[n]$ не перекрываются с орбиталями остальных его атомов. Это позволяет использовать приближение ближайших (или вторых, третьих и т.д.) соседей для матришы $Q_{1 / 2}[n]$.

Таким образом, вьшеперечисленные свойства матриц $(T[m])^{-1 / 2}$ и $Q_{1 / 2}[n]$ делают возможным проведение конкретных расчетов в низших порядках кластерного разложения.

Итак, при использовании разложения (7) выражение для $\Delta \psi_{\mathbf{k} s}(\mathbf{r})$ из (6) примет вид

$$
\begin{aligned}
\Delta \psi_{\mathbf{k} s}(\mathbf{r}) & =-\sum_{n=2}^{N} \sum_{[n] \subseteq[N]} \psi_{\mathbf{k} s}^{[n]}(\mathbf{r}), \\
\psi_{\mathbf{k} s}^{[n]}(\mathbf{r}) & =N^{-1 / 2} \sum_{\mathbf{l} \in[n]} \exp (i \mathbf{k} \mathbf{l}) \sum_{\mathbf{l}^{\prime} \in[n], s^{\prime}} \varphi_{\mathbf{1}^{\prime} s^{\prime}}(\mathbf{r}) Q_{1 / 2}[n]_{\mathbf{l}^{\prime} s^{\prime} ; \mathbf{l} s} .
\end{aligned}
$$

Слагаемое с $n=1$ в $(9)$ равно нулю, т.к. $Q_{1 / 2}[1]=\mathscr{P}[1]$ является нулевой матрицей. По той же причине в определяюшем матрицу $Q_{1 / 2}[n]$ разложении (7) суммирование по $m$ можно начинать с $m=2$.

Отметим некоторые свойства кластерных функций $\psi_{\mathbf{k} s}^{[n]}(\mathbf{r})$ :

1) функция $\psi_{\mathbf{k} s}^{[n]}(\mathbf{r})$ определяется только орбиталями $n$-частичного кластера и обрашается в нуль, если орбитали хотя бы одного из атомов кластера $[n]$ не перекрываются с орбиталями остальных его атомов. Последнее прямо следует из свойства 2 матрицы $Q_{1 / 2}[n]$ и выражения $(9)$;

2) используя трансляционную симметрию кристалла, можно показать (см. приложение 2), что

$$
\psi_{\mathbf{k} s}^{[n]}(\mathbf{r}+\mathbf{a})=\psi_{\mathbf{k} s}^{[n,-\mathbf{a}]}(\mathbf{r}) \exp (i \mathbf{k a}),
$$

где $[n,-\mathbf{a}] \equiv\left[\mathbf{l}_{1}-\mathbf{a} \ldots \mathbf{l}_{n}-\mathbf{a}\right]-$ кластер, сдвинутый относительно исходного $[n]$ на вектор решетки а. Функция

$$
\psi_{\mathbf{k} s}^{n}(\mathbf{r})=\sum_{[n] \subseteq[N]} \psi_{\mathbf{k} s}^{[n]}(\mathbf{r})
$$

является блоховской, т.е. $\psi_{\mathbf{k} s}^{n}(\mathbf{r}+\mathbf{a})=\psi_{\mathbf{k} s}^{n}(\mathbf{r}) \exp (i \mathbf{k a})$.

Подставляя (9) в $\left\langle\psi_{\mathbf{k} s}|H| \psi_{\mathbf{k} s^{\prime}}\right\rangle$, получим кластерное разложение для коэффициентов системы уравнений (6)

$$
\begin{aligned}
\left\langle\psi_{\mathbf{k} s}|H| \psi_{\mathbf{k} s^{\prime}}\right\rangle= & \left\langle\psi_{\mathbf{k} s}^{(0)}|H| \psi_{\mathbf{k} s^{\prime}}^{(0)}\right\rangle-\sum_{n=2}^{N} \sum_{[n] \subseteq[N]}\left\{\left\langle\psi_{\mathbf{k} s}^{[n]}|H| \psi_{\mathbf{k} s^{\prime}}^{(0)}\right\rangle+\left\langle\psi_{\mathbf{k} s}^{(0)}|H| \psi_{\mathbf{k} s^{\prime}}^{[n]}\right\rangle+\right. \\
& \left.+\sum_{n^{\prime}=2}^{N} \sum_{\left[n^{\prime}\right] \subseteq[N]}\left\langle\psi_{\mathbf{k} s}^{[n]}|H| \psi_{\mathbf{k} s^{\prime}}^{\left[n^{\prime}\right]}\right\rangle\right\} .
\end{aligned}
$$


В кристалле с одним атомом на ячейку волновые функции $\psi_{\mathbf{k} s}$ можно выбрать действительными. Тогда коэффициенты из (6) будут симметричны по $s$ и $s^{\prime}$.

Решая секулярное уравнение

$$
\operatorname{det}\left\|\left\langle\psi_{\mathbf{k} s}|H| \psi_{\mathbf{k} s^{\prime}}\right\rangle-E_{\mathbf{k} v} \delta_{s s^{\prime}}\right\|=0
$$

с коэффициентами $\left\langle\psi_{\mathbf{k} s}|H| \psi_{\mathbf{k} s^{\prime}}\right\rangle$ в приближении двухчастичных, трехчастичных и т.д. кластеров, можно вычислить зонную энергию электрона с требуемой точностью.

\section{4. МЕТОД СЕОРW}

Описанный выше метод CELO эффективен при расчете энергетических спектров кристаллов с сильно связанными электронами, а также остовных состояний металлов и полупроводников при высоких давлениях. Для расчета верхних валентных зон последних, а также зон проводимости сжатых кристаллов предлагается модифицированный метод OPW. Все дальнейшее изложение проведем на примере зон проводимости кристалла с сильной связью.

В предлагаемом подходе для построения блоховских функций $\Psi_{\mathbf{k c}}(\mathbf{r})$ зоны проводимости с индексом с используются плоские волны, ортогонализованные к волновым функциям заполненных зон кристалла (в данной работе мы не делаем разделения на валентные и остовные состояния),

$$
\begin{aligned}
& \Psi_{\mathbf{k} \mathfrak{c}}(\mathbf{r})=\phi_{\mathbf{k} \mathfrak{c}}(\mathbf{r})-\sum_{v, \mathbf{k}^{\prime}} \Psi_{\mathbf{k}^{\prime} v}(\mathbf{r}) \int \Psi_{\mathbf{k}^{\prime} v}^{*}\left(\mathbf{r}^{\prime}\right) \phi_{\mathbf{k} \mathfrak{c}}\left(\mathbf{r}^{\prime}\right) d \mathbf{r}^{\prime} \\
& \phi_{\mathbf{k} \mathfrak{c}}(\mathbf{r})=V^{-1 / 2} \sum_{\mathbf{g}} a_{\mathbf{k}}(\mathbf{g}) \exp (-i(\mathbf{k}+\mathbf{g}) \mathbf{r})
\end{aligned}
$$

Здесь $a_{\mathbf{k}}(\mathbf{g})$ - вариационные параметры, $\Psi_{\mathbf{k} v}(\mathbf{r})$ - блоховские функции заполненных зон кристалла, точно ортогонализованные друг к другу (см. (1)-(3)), g- вектор обратной решетки. Заметим, что ортогонализация $\Psi_{\mathbf{k c}}$ к волновым функциям занятых состояний является точной, если последние образуют точно ортонормированньй набор, что не соблюдается в традиционном методе OPW [1].

Подставляя (1) в (4) и вводя обозначение (cp. с (3))

$$
\varphi_{v}\left(\mathbf{r}-\mathbf{l}^{\prime}\right)=\sum_{s} \varphi_{s}\left(\mathbf{r}-\mathbf{l}^{\prime}\right) C_{s v},
$$

представим $\Psi_{\mathbf{k} v}$ в виде, аналогичном (6) и удобном для дальнейшей работы:

$$
\begin{aligned}
& \Psi_{\mathbf{k} v}(\mathbf{r})=N^{-1 / 2} \sum_{\mathbf{l}} \exp (i \mathbf{k} \mathbf{l})\left[\varphi_{v}(\mathbf{r}-\mathbf{l})-\sum_{\mathbf{l}^{\prime} v^{\prime}} \varphi_{v^{\prime}}\left(\mathbf{r}-\mathbf{l}^{\prime}\right) \Pi_{\mathbf{l}^{\prime} v^{\prime} ; \mathbf{l}}\right] \\
& \Pi_{\mathbf{l}^{\prime} v^{\prime} ; \mathbf{l} v}=\delta_{\mathbf{l}^{\prime}} \delta_{v v^{\prime}}-\sum_{s s^{\prime}} C_{s^{\prime} v^{\prime}}^{*}\left(T^{-1 / 2}\right)_{\mathbf{l}^{\prime} s^{\prime} ; \mathbf{l} s} C_{s v},
\end{aligned}
$$

где $C_{s v}$ - вариационные параметры метода CELO, которые считаются уже известными. 
По определению функции $\varphi_{v}(\mathbf{r}-\mathbf{l})$ неизменны при изменении межатомного расстояния. Функции $\Psi_{\mathbf{k} v} \quad(13)$ зависят от давления только через изменение интегралов перекрытия. Используя пробные фунцкии (12) с учетом (13), для коэффициентов $a_{\mathbf{k}}(\mathbf{g})$ метода CEOPW получаем следующую систему уравнений:

$$
\sum_{\mathbf{g}^{\prime}}\left\{H_{\mathbf{g g}^{\prime}}(\mathbf{k})-E_{\mathbf{k} \mathfrak{c}} \delta_{\mathbf{g g}^{\prime}}\right\} a_{\mathbf{k}}\left(\mathbf{g}^{\prime}\right)=0
$$

где

$$
H_{\mathbf{g g}^{\prime}}(\mathbf{k})=\frac{\hbar^{2}}{2 m}(\mathbf{k}+\mathbf{g})^{2} \delta_{\mathbf{g g}^{\prime}}+V_{C}\left(\mathbf{g}-\mathbf{g}^{\prime}\right)+V_{\mathrm{ex}}\left(\mathbf{k}+\mathbf{g} ; \mathbf{k}+\mathbf{g}^{\prime}\right)+V_{\mathrm{PK}}\left(\mathbf{k}+\mathbf{g} ; \mathbf{k}+\mathbf{g}^{\prime}\right)
$$

Здесь

$$
V_{C}(\mathbf{q})=\frac{4 \pi e^{2}}{q^{2} \Omega_{0}}(\rho(\mathbf{q})-Z)
$$

- формфактор кулоновского взаимодействия избыточного электрона с остальными электронами кристалла; $\rho(\mathbf{q})$ - фурье-образ электронной плотности

$$
\begin{gathered}
\rho(\mathbf{r})=2 \sum_{\mathbf{l} s}\left\{\varphi_{s}^{*}(\mathbf{r}-\mathbf{l}) \varphi_{s}(\mathbf{r}-\mathbf{l})-\sum_{\mathbf{l}^{\prime} s^{\prime}} \varphi_{s}(\mathbf{r}-\mathbf{l}) P_{\mathbf{l} s ; \mathbf{1}^{\prime} s^{\prime}} \varphi_{s^{\prime}}^{*}\left(\mathbf{r}-\mathbf{l}^{\prime}\right)\right\}, \\
s=(n, L, m), \quad P=I-T^{-1} .
\end{gathered}
$$

Суммирование в (16) ведется не по заполненным зонам $v$, а по атомным состояниям $s$, т.к. в силу унитарности матрицы $C$ вариационных параметров $\rho=\sum_{\mathbf{k} v} \Psi_{\mathbf{k} v} \Psi_{\mathbf{k} v}^{*}=$ $\sum_{\mathbf{k} s} \Psi_{\mathbf{k} s} \Psi_{\mathbf{k} s}^{*}$

Слагаемое $V_{\text {ex }}\left(\mathbf{q} ; \mathbf{q}^{\prime}\right)$ в (14) - формфактор обменного взаимодействия избыточного электрона с остальными:

$$
\begin{aligned}
V_{\mathrm{ex}}\left(\mathbf{q} ; \mathbf{q}^{\prime}\right)= & -\frac{e^{2}}{2 \pi^{2}} \sum_{v} \int \frac{d^{3} \varkappa}{\varkappa^{2}}\left\{I_{v}^{*}(\mathbf{q}-\varkappa) I_{v}\left(\mathbf{q}^{\prime}-\varkappa\right)-\right. \\
& -\left[I_{v}^{*}(\mathbf{q}-\varkappa) \sum_{v^{\prime}} I_{v^{\prime}}\left(\mathbf{q}^{\prime}-\varkappa\right) \Pi_{v^{\prime} v}^{*}(\mathbf{q}-\varkappa)+\text { э.c. }\right]+ \\
& \left.\sum_{v^{\prime} v^{\prime \prime}} I_{v^{\prime}}^{*}(\mathbf{q}-\varkappa) I_{v^{\prime \prime}}\left(\mathbf{q}^{\prime}-\varkappa\right) \Pi_{v^{\prime} v}(\mathbf{q}-\varkappa) \Pi_{v^{\prime \prime} v}^{*}\left(\mathbf{q}^{\prime}-\varkappa\right)\right\} .
\end{aligned}
$$

Слагаемое $V_{\mathrm{PK}}\left(\mathbf{q} ; \mathbf{q}^{\prime}\right)$ - формфактор псевдопотенциала Филипса-Клейнмана:

$$
\begin{aligned}
V_{\mathrm{PK}}\left(\mathbf{q} ; \mathbf{q}^{\prime}\right)= & \sum_{v}\left(E_{\mathbf{k} \mathfrak{c}}-E_{\mathbf{k} v}\right)\left\{I_{v}^{*}(\mathbf{q}) I_{v}\left(\mathbf{q}^{\prime}\right)-\left[I_{v}^{*}(\mathbf{q}) \sum_{v^{\prime}} I_{v^{\prime}}\left(\mathbf{q}^{\prime}\right) \Pi_{v^{\prime} v}^{*}\left(\mathbf{q}^{\prime}\right)+\text { э.c. }\right]+\right. \\
& \left.+\sum_{v^{\prime} v^{\prime \prime}} I_{v^{\prime}}^{*}(\mathbf{q}) I_{v^{\prime \prime}}\left(\mathbf{q}^{\prime}\right) \Pi_{v^{\prime} v}(\mathbf{q}) \Pi_{v^{\prime \prime} v}^{*}\left(\mathbf{q}^{\prime}\right)\right\} .
\end{aligned}
$$


В формулах (17) и (18) $I_{v}(\mathbf{q})$ - фурье-образ локализованной волновой функции $\varphi_{v}^{*}(\mathbf{r})[2-4]$,

$$
\Pi_{v^{\prime} v}(\mathbf{q})=\sum_{\mathbf{l}} \Pi_{\mathbf{l} v^{\prime} ; \mathbf{0} v} \exp (i \mathbf{q} \mathbf{l})
$$

Можно показать, что $\Pi_{v^{\prime} v}(\mathbf{q})=\Pi_{v v^{\prime}}^{*}(\mathbf{q})$. Если волновые функции электронов атома выбраны вешественными, то $\Pi_{v^{\prime} v}(\mathbf{q})$ симметричны по $v$ и $v^{\prime}$. Напомним, что при нахождении зон проводимости $E_{\mathbf{k c}}$ законы дисперсии валентных зон $E_{\mathbf{k} v}$ считаются известными.

Таким образом, мы получили выражение для составляющих псевдогамильтоновой матрицы в (14), используя точно ортогонализованные друг к другу волновые функции (4) (или (13)) занятых состояний кристалла и плоские волны, точно ортогонализованные к занятым состояниям. Сравнивая потенциалы с точной ортогонализацией $V_{\text {ex }}$ (17) и $V_{\mathrm{PK}}(18)$ с соответствуюшими "приближенными" из [2-4], можно заметить, что последние отличаются от (17) и (18) заменой в них (потенциалах из [2-4]) $S_{v v^{\prime}}$ на П $\boldsymbol{v}^{\prime} v$ и добавлением дополнительных слагаемых, квадратичных по $\Pi_{v^{\prime} v}(\mathbf{q})$. Расчету $\Pi_{v^{\prime} v}(\mathbf{q})$ посвящена оставшаяся часть данной работы.

Точная ортогонализация приводит к появлению в кулоновском потенциале (15)-(16) принципиально нового слагаемого (последнее слагаемое в (16)), не имеющего аналога в [2-4]. Методика расчета данного слагаемого приведена в [8-10].

\section{5. КЛАСТЕРНОЕ РАЗЛОЖЕНИЕ В МЕТОДЕ СЕОРШ}

Кластерное разложение для матрицы $\Pi_{v^{\prime} v}(\mathbf{k})$ имеет вид

$$
\begin{aligned}
& \Pi_{v^{\prime} v}(\mathbf{k})=\sum_{n=2}^{N} \Pi_{v^{\prime} v}^{(n)}(\mathbf{k}) \\
& \Pi_{v^{\prime} v}^{(n)}(\mathbf{k})=\sum_{\left[n_{0}\right] \subseteq[N]} \sum_{\mathbf{l} \in\left[n_{0}\right]} \exp (i \mathbf{k} \mathbf{l}) Q_{1 / 2}\left[n_{0}\right]_{\mathbf{l} v^{\prime} ; \mathbf{0} v} .
\end{aligned}
$$

Здесь через $\left[n_{0}\right] \equiv\left[\mathbf{l}_{1} \ldots \mathbf{0} \ldots \mathbf{l}_{n}\right]$ обозначен $n$-частичный кластер, содержаший нулевой узел решетки. Величина $Q_{1 / 2}\left[n_{0}\right]$ определена ранее. Подставляя (19) в (17) и (18), получаем следующие кластерные разложения для потенциалов:

$$
\begin{aligned}
V_{\mathrm{ex}}\left(\mathbf{q} ; \mathbf{q}^{\prime}\right)= & -\frac{e^{2}}{2 \pi^{2}} \sum_{n=2}^{N} \sum_{v} \int \frac{d^{3} \varkappa}{\varkappa^{2}}\left\{I_{v}^{*}(\mathbf{q}-\varkappa) I_{v}\left(\mathbf{q}^{\prime}-\varkappa\right)-\right. \\
& -\left[I_{v}^{*}(\mathbf{q}-\varkappa) \sum_{v^{\prime}} I_{v^{\prime}}\left(\mathbf{q}^{\prime}-\varkappa\right) \Pi_{v^{\prime} v}^{(n) *}\left(\mathbf{q}^{\prime}-\varkappa\right)+\ni . c .\right]+ \\
& \left.+\sum_{n^{\prime}=2}^{N} \sum_{v^{\prime} v^{\prime \prime}} I_{v^{\prime}}^{*}(\mathbf{q}-\varkappa) I_{v^{\prime \prime}}\left(\mathbf{q}^{\prime}-\varkappa\right) \Pi_{v^{\prime} v}^{\left(n^{\prime}\right)}(\mathbf{q}-\varkappa) \Pi_{v^{\prime \prime} v}^{(n) *}\left(\mathbf{q}^{\prime}-\varkappa\right)\right\}
\end{aligned}
$$




$$
\begin{aligned}
V_{\mathrm{PK}}\left(\mathbf{q}, \mathbf{q}^{\prime}\right)= & \sum_{n=2} \sum_{v}\left(E_{\mathbf{k} \mathfrak{c}}-E_{\mathbf{k} v}\right)\left\{I_{v}^{*}(\mathbf{k}+\mathbf{g}) I_{v}\left(\mathbf{k}+\mathbf{g}^{\prime}\right)-\right. \\
& -\left[I_{v}^{*}(\mathbf{q}) \sum_{v^{\prime}} I_{v^{\prime}}\left(\mathbf{q}^{\prime}\right) \Pi_{v^{\prime} v}^{(n) *}\left(\mathbf{q}^{\prime}\right)+\text { э.c. }\right]+ \\
& \left.+\sum_{n^{\prime}=2} \sum_{v^{\prime} v^{\prime \prime}} I_{v^{\prime}}^{*}(\mathbf{q}) I_{v^{\prime \prime}}\left(\mathbf{q}^{\prime}\right) \Pi_{v^{\prime} v}^{\left(n^{\prime}\right)}(\mathbf{q}) \Pi_{v^{\prime \prime} v}^{(n) *}\left(\mathbf{q}^{\prime}\right)\right\} .
\end{aligned}
$$

Эти выражения отличаются от (17) и (18) тем, что в них вместо $\Pi_{v^{\prime} v}(\mathbf{k})$ стоит $\Pi_{v^{\prime} v}^{(n)}(\mathbf{k})$ и ведется суммирование по всем $n$.

Формфактор кулоновского потенциала $V_{C}\left(\mathbf{g}-\mathbf{g}^{\prime}\right)$ зависит от электронной плотности $\rho(\mathbf{r})$, кластерное разложение для которой приведено в [8-10]. С учетом этого разложения $V_{C}\left(\mathbf{g}-\mathbf{g}^{\prime}\right)$ принимает вид

$$
V_{C}(\mathbf{q})=\frac{4 e^{2} \pi}{q^{2} \Omega_{0}}\left\{\rho^{(0)}(\mathbf{q})-\sum_{n=2}^{N} \rho(n)(\mathbf{q})-Z\right\},
$$

где при $n=0,2,3,4, \ldots$ :

$$
\begin{aligned}
\rho^{(n)}(\mathbf{q}) & =N^{-1} \int \rho^{(n)}(\mathbf{r}) \exp (-i \mathbf{q r}) d \mathbf{r}, \\
\rho^{(n)}(\mathbf{r}) & =2 \sum_{[n] \subseteq[N]} \sum_{\mathbf{l l}^{\prime} \in[n], s s^{\prime}} \varphi_{s}(\mathbf{r}-\mathbf{l}) Q_{1}[n]_{\mathbf{l} s ; \mathbf{1}^{\prime} s^{\prime}} \varphi_{s^{\prime}}^{*}\left(\mathbf{r}-\mathbf{l}^{\prime}\right), \\
Q_{1}\left[\mathbf{l}_{1} \ldots \mathbf{l}_{n}\right] & =-\sum_{m=2}^{n} \sum_{[m] \subseteq[n]}(-1)^{m+n}\left(I-(T[m])^{-1}\right) .
\end{aligned}
$$

\section{6. ПРИБЛИЖЕНИЕ ДВУХЧАСТИЧНЫХ КЛАСТЕРОВ}

В предыдущих разделах были получены кластерные разложения для потенциалов. Однако с увеличением числа $n$ частиц в кластере объем расчетов резко возрастает, т.к. приходится диагонализировать или обращать (см. (7) и (16)) матрицы все большего порядка (равного $(Z / 2) \cdot n)$. Однако эта проблема вполне разрешима, поскольку для практических целей, по-видимому, нет смысла использовать кластеры, состоящие более чем из трех частиц [10]. Мы здесь рассмотрим только приближение двухчастичных кластеров (two-particle cluster - TPC). Это приближение, кроме того, дает возможность для метода CEOPW осуществить предельный переход к результатам [2-4].

Приближение TPC для метода CELO получается, если в кластерном разложении (9) для элементов псевдогамильтоновой матрищы оставить слагаемые с $n, n^{\prime}$ до двух включительно:

$$
\begin{aligned}
\left\langle\psi_{\mathbf{k} s}|H| \psi_{\mathbf{k} s^{\prime}}\right\rangle= & \left\langle\psi_{\mathbf{k} s}^{(0)}|H| \psi_{\mathbf{k} s^{\prime}}^{(0)}\right\rangle-\sum_{\mathbf{l}<\mathbf{l}^{\prime}}\left\{\left[\left\langle\psi_{\mathbf{k} s}^{\left[\mathbf{l 1}^{\prime}\right]}|H| \psi_{\mathbf{k} s^{\prime}}^{(0)}\right\rangle+\text { э.c. }\right]+\right. \\
& \left.+\sum_{\mathbf{n}<\mathbf{n}^{\prime}}\left\langle\psi_{\mathbf{k} s}^{\left[\mathbf{l 1}^{\prime}\right]}|H| \psi_{\mathbf{k} s^{\prime}}^{\left[\mathbf{n} \mathbf{n}^{\prime}\right]}\right\rangle\right\},
\end{aligned}
$$


Если в (24) пренебречь квадратичными по $\psi_{\mathbf{k} s}^{\left[\mathbf{l 1}^{\prime}\right]}(\mathbf{r})$ слагаемыми, то система уравнений (4) принимает вид

$$
\begin{gathered}
\sum_{s^{\prime}}\left\{\left(\varepsilon_{s}-E_{\mathbf{k} v}\right) \delta_{s s^{\prime}}+V_{s s^{\prime}}^{(0 a)}(\mathbf{k})+V_{s s^{\prime}}^{(0 b)}(\mathbf{k})+V_{s s^{\prime}}^{(1 a)}(\mathbf{k})+\right. \\
\left.+V_{s s^{\prime}}^{(1 b)}(\mathbf{k})+V_{s s^{\prime}}^{(1 c)}(\mathbf{k})\right\} C_{s^{\prime} v}=0
\end{gathered}
$$

где

$$
V_{s s^{\prime}}^{(x y)}(\mathbf{k})=\sum_{\mathbf{l}} \exp (i \mathbf{k} \mathbf{l}) V_{s s^{\prime}}^{(x y)}(\mathbf{l}), \quad x=0,1, \quad y=a, b, c .
$$

Слагаемые с $x=0$, имеющие нулевой порядок по $\mathscr{P}\left[\mathbf{l 1}^{\prime}\right]$, равны

$$
\begin{aligned}
V_{s s^{\prime}}^{(0 a)}(\mathbf{l}) & =\sum_{\mathbf{l}^{\prime} \neq \mathbf{0}, \mathbf{l}}\left\langle\varphi_{\mathbf{0} s}\left|v_{\mathbf{l}^{\prime}}\right| \psi_{\mathbf{1} s^{\prime}}\right\rangle, \\
V_{s s^{\prime}}^{(0 b)}(\mathbf{l}) & =\left(\left\langle\varphi_{\mathbf{0} s}\left|v_{\mathbf{0}}\right| \psi_{\mathbf{l} s^{\prime}}\right\rangle+\left\langle\varphi_{\mathbf{0} s}\left|v_{\mathbf{l}}\right| \psi_{\mathbf{l} s^{\prime}}\right\rangle\right) \times\left(1-\delta_{\mathbf{0} \mathbf{l}}\right) .
\end{aligned}
$$

При $\mathbf{l}=\mathbf{0}$ в представлении (27) содержатся двухцентровые интегралы, в противном случае - трехцентровые. В представлении (28) содержатся только двухцентровые интегралы.

Слагаемые с $x=1$, имеюшие первый порядок по $\mathscr{P}\left[\mathbf{1 1}^{\prime}\right]$, равны

$$
\begin{aligned}
& V_{s s^{\prime}}^{(1 y)}(\mathbf{l})=\sum_{s^{\prime \prime} \mathbf{1}^{\prime \prime}}\left\{V_{s s^{\prime \prime}}^{(0 y)}\left(\mathbf{l}^{\prime \prime}\right) \mathscr{P}\left[\mathbf{l}^{\prime \prime}\right]_{\mathbf{1}^{\prime \prime} s^{\prime \prime} ; \mathbf{l} s^{\prime}}+\right. \\
& \left.+V_{s s^{\prime \prime}}^{(0 y)}(\mathbf{l}) \mathscr{P}\left[\mathbf{l l}^{\prime \prime}\right]_{\mathbf{l} s^{\prime \prime} ; \mathbf{l} s^{\prime}}\right\}+ \text { Э.c., } \quad y=a, b, \\
& V_{s s^{\prime}}^{(1 c)}(\mathbf{k})=\varepsilon_{s}\left\{\mathscr{P}[\mathbf{0 1}]_{\mathbf{0} s ; \mathbf{l} s^{\prime}}+\mathscr{P}[\mathbf{0 1}]_{\mathbf{0} s ; \mathbf{0} s^{\prime}} \delta_{\mathbf{k} \mathbf{0}}\right\}+\text { э.c. }
\end{aligned}
$$

Напомним, что в (26)-(30) $\varphi_{\mathbf{l} s}$ являются собственными функциями уравнения Шредингера (2), а $\varepsilon_{s}$ - его собственными значениями.

Таким образом, для решения системы (25) (т.е. для нахождения ее коэффициентов (35)-(39)) необходимо рассчитать двух- и трехцентровые интегралы, содержашиеся в $V_{s s^{\prime}}^{(0 a)}(\mathbf{l})(27)$ и в $V_{s s^{\prime}}^{(0 b)}(\mathbf{l})(28)$, и элементы кластерной матрицы $\mathscr{P}\left[\mathbf{1}^{\prime \prime}\right]$. Способ расчета последних дан в приложении 1.

Приближение двухчастичных кластеров для $V_{\mathrm{ex}}, V_{\mathrm{PK}}$ и $V_{c}$ метода CEOPW получается, если в выражениях (19)-(22) оставить слагаемые с $n, n^{\prime}=2$. Выражения для $V_{\mathrm{ex}}^{(2)}$, $V_{\mathrm{PK}}^{(2)}$ и $V_{c}^{(2)}$ в приближении ТРС выглядят аналогично соответствуюшим выражениям с точной ортогонализацией (15)-(19), только в ТРС вместо $\Pi_{v^{\prime} v}(\mathbf{k})$ и $\rho(\mathbf{k})$ стоят $\Pi_{v^{\prime} v}^{(2)}(\mathbf{k})$ и $\rho^{(2)}(\mathbf{k})$, причем (см. (19) при $\left.n=2\right)$

$$
\Pi_{v^{\prime} v}^{(2)}(\mathbf{k})=\sum_{\mathbf{l} \neq \mathbf{0}}\left\{\exp (i \mathbf{k} \mathbf{l}) \Pi[\mathbf{0 1}]_{\mathbf{l} v^{\prime} ; \mathbf{0} v}+\Pi[\mathbf{0} \mathbf{l}]_{\mathbf{0} v^{\prime} ; \mathbf{0} v}\right\}
$$

где (см. (13))

$$
\Pi[\mathbf{0 l}]=I-C^{-1}(I+S[\mathbf{0 l}])^{-1 / 2} C,
$$


a $\rho^{(2)}\left(\mathbf{g}^{\prime}-\mathbf{g}\right)$ определяется формулой $(23)$ при $n=2$.

С учетом (П1.5) выражение (31) для $\Pi_{v^{\prime} v}^{(2)}(\mathbf{k})$ примет вид

$$
\begin{aligned}
\Pi_{v^{\prime} v}^{(2)}(\mathbf{k})= & \delta_{v v^{\prime}}-\sum_{\mathbf{l} \neq \mathbf{0}} \sum_{j=j_{1}}^{j_{10}}\left\{\sum_{s s^{\prime}} C_{s^{\prime} v^{\prime}}^{*} \frac{X[\mathbf{0 1}]_{\mathbf{0}, s^{\prime}}^{(j)} X[\mathbf{0 1}]_{\mathbf{0}, s}^{*(j)}}{\left(\boldsymbol{\mu}^{(j)}[\mathbf{0 1}]\right)^{1 / 2}} C_{s v}-\right. \\
& \left.-\sum_{s s^{\prime}} C_{s^{\prime} v^{\prime}}^{*} \frac{X[\mathbf{0 1}]_{\mathbf{l}, s^{\prime}}^{(j)} X[\mathbf{0 1}]_{\mathbf{0}, s}^{*(j)}}{\left(\mu^{(j)}[\mathbf{0 l}]\right)^{1 / 2}} C_{s v} \exp (i \mathbf{k} \mathbf{l})\right\},
\end{aligned}
$$

где $X_{\mathbf{l} s^{\prime}}^{(j)}[\mathbf{0 l}]$ и $\mu^{(j)}[\mathbf{0 1}]$ выражаются через интегралы перекрытия (2) волновых функций $\varphi_{s}(\mathbf{r}-\mathbf{l})$. Формула (32) решает задачу о вычислении элементов псевдогамильтоновой матрицы $V_{\mathrm{ex}}^{(2)}, V_{\mathrm{PK}}^{(2)}$ и $V_{c}^{(2)}$ в приближении ТРС и в конечном итоге дает решение системы уравнений (15) метода CEOPW.

\section{7. ЗАКЛЮЧЕНИЕ}

Простейший путь учета ортогонализационных поправок - разложить матрицы $P$ в (16) и П в $(17),(18)$ в ряд по матрице $S \equiv T-I$ и ограничиться низшими порядками. Например, если оставить в матришах П и $P$ слагаемые, линейные по $S$, и использовать приближение ближайших соседей, то выражения (16)- (18) сводятся к полученным в [2], что соответствует ортогонализации по Шмидту. Однако параметр, описываюший влияние соседей на волновую функцию выделенного атома, как уже отмечалось, равен $|S| z$ и при больших сжатиях становится порядка единицы.

Таким образом, при исследовании кристаллов под давлением целесообразно воспользоваться кластерным разложением матрищ $P$ и П, которое эквивалентно перегруппировке членов бесконечных рядов и их частичному суммированию (см. $[9,7])$. Подчеркнем, что при выводе формул кластерного разложения степенные ряды по $|S|$ не использовались и кластерное разложение справедливо при любых давлениях (интегралах перекрытия).

Для того чтобы оценить члены кластерных разложений (7) для методов CELO и CEOPW, оценим матрицы $Q_{t}[n], t=1,1 / 2$. Разложим матрицы $(T[m])^{-t}$ в ряд по $S[m](8)$. Матрица $S[m]$ имеет порядок величины $|S|$ в том смысле, что наибольшие из ее элементов $\sim|S|$. Разложения по $S[m]$ для матриц $Q_{t}$ из $(19)$ и $(23)$ примут вид

$$
\begin{aligned}
Q_{t}[n] & =\sum_{m=1}^{n} \sum_{[m] \subseteq[n]}(-1)^{m+n} \sum_{i=1}^{\infty}(-1)^{i} B_{i}^{t}(S[m])^{i} \\
B_{i}^{t} & = \begin{cases}\frac{\frac{1}{2} \cdot \frac{3}{2} \cdots\left(i-\frac{1}{2}\right)}{i !}, & t=1 / 2, \\
1, & t=1 .\end{cases}
\end{aligned}
$$

Несмотря на то что разложения по $S[m]$ для $Q_{1}[n]$ и $Q_{1 / 2}[n]$ несколько отличаются друг от друга, все последуюшие выводы справедливы для обеих матриц. Используя доказательство, приведенное в [10] для $Q_{1}[n]$ и справедливое для $Q_{1 / 2}[n]$ (т.к. разложения по $S[m]$ для $Q_{1}[n]$ и $Q_{1 / 2}[n]$ отличаются друг от друга лишь коэффициентами), получим, 
что для диагональных блоков $Q_{t}[n]_{\mathbf{1} ; \mathbf{l}}(t=1,1 / 2)$ ненулевые слагаемые начинаются с $i=n$, а для недиагональных блоков $Q_{t}[n]_{\mathbf{1} ; \mathbf{1}^{\prime}}-$ с $i=n-1$. Разложение по $S[m]$ для матриц $Q_{t}[n]$ содержит также слагаемые и более высоких порядков по $|S|$ ("хвост"). При сильных сжатиях обсуждаемые ряды могут медленно сходиться по $i$, так что вклад "хвоста" ряда по сравнению с главным слагаемым неясен до выполнения численного расчета. По той же причине трудно оценить, насколько недиагональные блоки матрицы $Q_{t}[n]_{\mathbf{1} ; \mathbf{1}^{\prime}}$ больше диагональных.

Подставляя разложения по $S[\mathrm{~m}]$ для $Q_{t}$ в соответствуюшие кластерные разложения для элементов псевдогамильтоновых матрищ методов CELO и CEOPW, можно аналогичным образом оценить ортогонализационные поправки к этим величинам, обусловленные $n$-частичными кластерами. Таким образом, если оборвать указанные кластерные разложения на конечном $n$ с целью получить требуемую точность, то степень интеграла перекрытия $|S|$ при высоких давлениях не будет подходяшим параметром, говорящим о точности полученного приближения. Необходимо всегда помнить о "хвостах" рядов по степеням $S[m]$.

Расчеты зон обычно выполняются в нулевом приближении по параметру адиабатичности $\eta=\hbar \omega_{\mathrm{D}} / E_{g}\left(\omega_{\mathrm{D}}-\right.$ дебаевская частота, $E_{g}$ - ширина запрешенной зоны) [11]. При повышении давления ширина зонной шели будет уменьшаться $\left(E_{g} \rightarrow 0\right)[3,4]$ и $\eta$ станет порядка единищы. В этом случае фононы начнут играть активную роль в формировании зонной структуры, но предложенный подход не потеряет актуальности. Действительно, давление $p_{c}$ перехода "диэлектрик-металл" велико, а его изменение $\delta p_{c}$ за счет электрон-фоононных процессов есть

$$
\left(\delta p_{c}\right) / p_{c} \sim \frac{\hbar \omega_{\mathrm{D}}\left(p_{c}\right)}{p_{c}\left(d E_{g} / d p\right)_{p_{c}}} \sim 10^{-4}
$$

(по оценкам из расчетов зонной структуры $[4,12]$ и теории перехода “диэлектрик-металл" [13] $\hbar \omega_{\mathrm{D}}\left(p_{c}\right) \sim 5 \cdot 10^{-3}{ }_{\ni} \mathrm{B}, p_{c}\left(d E_{g} / d p\right)_{p} \sim 20$ эВ). Таким образом, учитьвая точность определения $p_{c}[4]$, влиянием неадиабатичности в точке перехода $p_{c}$ можно пренебречь.

Следующим приближением, лежащим в основе настоящей работы, является одноэлектронное приближение. Для молекулярных и ионных кристаллов, в которых эффекты корреляции слабы, одноэлектронное приближение работает хорошо. Для полупроводниковых кристаллов межэлектронные корреляции требуют дополнительного рассмотрения.

Авторы выражают глубокую благодарность И.В. Абаренкову за многочисленные обсуждения и внимание к работе. Эти исследования стали возможны частично благодаря гранту № U9B000 Международного научного фонда.

ПРИЛОЖЕНИЕ 1

\section{РАСЧЕТ МАТРИЦЫ $(T[m])^{-1 / 2}$}

Стандартная процедура извлечения корня из матрицы (см., например, $[10,14])$ включает в себя следуюшие этапы: 1) диагонализация исходной матрицы $T[m], 2)$ построение 
матрицы $W$ с элементами $W[m]_{j k}=\left|\left(\mu^{(j)}[m]\right)^{-1 / 2}\right| \cdot \delta_{j k}$, где $\mu^{(j)}[m]-$ собственные значения матрицы $T[m], 3)$ преобразование $(T[m])^{-1 / 2}=U[m] W[m]^{-1} U[m]^{-1}$, где $U[m]$ - матрица, построенная из собственных векторов матрицы $T[m](U[m]$ унитарна, т.к. $T[m]$ эрмитова). Матрицу $T[m]$ будем считать известной. Мы остановимся лишш на некоторых моментах, важных для данной работы. Сначала покажем, что для диагонализации матришы $T[m]$ на самом деле достаточно решить характеристическое уравнение порядка $m \cdot(Z / 2)(m$ - число атомов в кластере $)$, хотя на первый взгляд оно имеет порядок $N \cdot(Z / 2)$.

Если разложить детерминант в уравнении $\operatorname{det}\|\mu I-T[m]\|=0$ по строкам, состояшим из блоков типа II матришы $T[m]$, то оно примет вид

$$
(\mu-1)^{(N-m) Z / 2} \operatorname{det}\|\mu I-M[m]\|=0,
$$

где $M[m]$ - квадратная матрица порядка $(m \cdot(Z / 2))$, которая получается из матришы $T[m]$ вычеркиванием всех ее строк и столбцов, состоящих из блоков типа II. Кроме того, из (П.1.1) видно, что матрица $T[m]$ имеет $(N-m) \cdot(Z / 2)$ собственных значений, равных единице, и $m \cdot(Z / 2)$ - не равных ей. Таким образом, свойство 2 матрицы $(T[m])^{-1 / 2}$ доказано.

Для простоты рассмотрим двухчастичный кластер $[m] \equiv[\mathbf{0 1}]$ для кристалла неона (десять электронов на атом в конфигурации $1 s^{2} 2 s^{2} 2 p^{6}$ ). Получим в явном виде формулу для элементов $(T[\mathbf{0 1}])_{\mathbf{1}^{\prime} s: 1 s}^{-1 / 2}\left(s, s^{\prime}=1 s, 2 s, 2 p_{x}, 2 p_{y}, 2 p_{z}\right)$, из которой станет очевидным свойство 1 матрицы $(T[\mathbf{0 1}])^{1 / 2}$. Пусть матрица $T[\mathbf{0 1}]$ уже диагонализована, т.е. найдены ее собственные значения

$$
\mu^{(j)}[\mathbf{0 l}]= \begin{cases}\mu^{(j)}[\mathbf{0 l}], & j \in\left\{j_{1} \ldots j_{10}\right\}, \\ 1 & \text { в противном случае, }\end{cases}
$$

и векторы

$$
\begin{aligned}
X[\mathbf{0 1}]^{(j)}=\{ & 0, \ldots 0, X[\mathbf{0 1}]_{\mathbf{0}, 1 s}^{(j)}, \ldots, X[\mathbf{0 1}]_{\mathbf{0}, 2 p_{z}}^{(j)}, \\
& \left.X[\mathbf{0}]_{\mathbf{l}, 1 s}^{(j)}, \ldots, X[\mathbf{0 1}]_{\mathbf{1}, 2 p_{z}}^{(j)}, 0, \ldots, 0\right\}
\end{aligned}
$$

для $j$, соответствующих $\mu^{(j)}[\mathbf{0 l}] \neq 1$, и

$$
X[\mathbf{0 1}]_{\mathbf{1}^{\prime \prime}, s}^{(j)} \equiv X[\mathbf{0 1}]_{\mathbf{1}^{\prime \prime}, s}^{\left(\mathbf{1}^{\prime} s^{\prime}\right)}=\delta_{\mathbf{1}^{\prime} \mathbf{1}^{\prime \prime}} \delta_{s s^{\prime}}
$$

для $j$, соответствующих $\mu^{(j)}[\mathbf{0 l}]=1$.

С учетом (П.1.2)-(П.1.4) $(T[\mathbf{0 1}])_{\mathbf{1}^{\prime} s^{\prime} ; 1 s}^{-1 / 2}$ примут вид

$$
\begin{aligned}
& (T[\mathbf{0 1}])_{\mathbf{1}^{\prime} s^{\prime} ; \mathbf{1}^{\prime \prime} s}^{-1 / 2}=\sum_{j=1}^{L} \frac{X[\mathbf{0 1}]_{\mathbf{1}^{\prime}, s^{\prime}}^{(j)} X[\mathbf{0 1}]_{\mathbf{1}^{\prime \prime}, s}^{*(j)}}{\left(\mu^{j}[\mathbf{0 1}]\right)^{1 / 2}}= \\
& = \begin{cases}\sum_{j=j_{1}}^{j_{10}} \frac{X[\mathbf{0 1}]_{\mathbf{l}^{\prime}, s^{\prime}}^{(j)} X[0 \mathbf{0}]_{\mathbf{1}^{\prime \prime}, s}^{*(j)}}{\left(\mu^{(j)}[\mathbf{0 1}]\right)^{1 / 2}}, & \mathbf{1}^{\prime}, \mathbf{1}^{\prime \prime} \in[\mathbf{0 1}], \\
\delta_{\mathbf{1}^{\prime} \mathbf{1}^{\prime \prime}} \delta_{s s^{\prime}} & \text { в противном случае. }\end{cases}
\end{aligned}
$$

Из (П.1.5) очевидно свойство 1 матрицы $(T[\mathbf{0 1}])^{-1 / 2}$. Аналогичным способом это свойство доказывается для кластеров с произвольным числом частиц. 
ПРИЛОЖЕНИЕ 2

ТРАНСЛЯЦИОННЫЕ СВОЙСТВА ФУНКЦИИ $\psi_{\mathbf{k} s}^{[n]}(\mathbf{r}) \quad(9)$

Выясним, как ведет себя функция $\psi_{\mathbf{k} s}^{[n]}(\mathbf{r})$ при трансляции на вектор решетки $\mathbf{a}$,

$$
\begin{aligned}
\psi_{\mathbf{k} s}^{[n]}(\mathbf{r}+\mathbf{a}) & =N^{-1 / 2} \sum_{\mathbf{l} \in[n]} \exp (i \mathbf{k} \mathbf{l}) \sum_{\mathbf{l}^{\prime} \in[n], s^{\prime}} \varphi_{\mathbf{l}^{\prime} s^{\prime}}(\mathbf{r}+\mathbf{a}) Q_{1 / 2}[n]_{\mathbf{l}^{\prime} s^{\prime} ; \mathbf{l} s}= \\
& =\exp (i \mathbf{k a}) N^{-1 / 2} \sum_{\mathbf{l} \in[n]} \exp (i \mathbf{k}(\mathbf{l}-\mathbf{a})) \sum_{\mathbf{l}^{\prime} \in[n], s^{\prime}} \varphi_{\mathbf{l}^{\prime}-\mathbf{a}, s^{\prime}}(\mathbf{r}) Q_{1 / 2}[n]_{\mathbf{l}^{\prime} s^{\prime} ; \mathbf{l} s} .
\end{aligned}
$$

Рассмотрим свойства элементов матрицы $Q_{1 / 2}[n]$ кластера $[n]$, используя ее определение (см. (7) при $t=1 / 2)$. В силу трансляционной симметрии кристалла элементы полной метрической матрицы $T$ обладают свойством

$$
T_{\mathbf{l}^{\prime} s^{\prime} ; \mathbf{1} s}=T_{\mathbf{1}^{\prime} \pm \mathbf{a}, s^{\prime} ; \mathbf{1} \pm \mathbf{a}, s} .
$$

Следовательно, метрические матрицы $T[m]$ и $T[m, \pm \mathbf{a}]$ кластеров $[m] \equiv\left[\mathbf{l}_{1} \ldots \mathbf{l}_{m}\right]$ и $[m, \pm \mathbf{a}] \equiv\left[\mathbf{l}_{1} \pm \mathbf{a} \ldots \mathbf{l}_{m} \pm \mathbf{a}\right]$, фигурируюшие в (7) (а значит, и матрищы $(T[m])^{-1 / 2}$ и $\left.(T[m, \pm \mathbf{a}])^{-1 / 2}\right)$, будут иметь одинаковые элементы. Единственное отличие между матрицами, относящимися к указанным кластерам, состоит в перестановке строк и столбцов, которая при суммировании приводит лишь к перестановке слагаемых в сумме и не влияет на конечные результаты. Тогда у $Q_{1 / 2}[n]$ и $Q_{1 / 2}[n, \pm \mathbf{a}]$ будут также одинаковые элементы (в вышеупомянутом смысле) и любую сумму вида

$$
\sum_{\mathbf{l} \in[n]} \varphi_{\mathbf{l}, s^{\prime}}(\mathbf{r}) Q_{1 / 2}[n]_{\mathbf{1}^{\prime} s^{\prime} ; \mathbf{1} s}
$$

можно заменить на

$$
\sum_{\mathbf{l} \pm \mathbf{a} \in[n, \pm \mathbf{a}]} \varphi_{\mathbf{l}, s^{\prime}}(\mathbf{r}) Q_{1 / 2}[n, \pm \mathbf{a}]_{\mathbf{l}^{\prime}+\mathbf{a}, s^{\prime} ; \mathbf{l}+\mathbf{a}, s}
$$

Таким образом,

$$
\begin{aligned}
\psi_{\mathbf{k} s}^{[n]}(\mathbf{r}+\mathbf{a})= & \exp (i \mathbf{k a}) N^{-1 / 2} \sum_{\mathbf{l}-\mathbf{a} \in[n,-\mathbf{a}]} \exp (i \mathbf{k}(\mathbf{l}-\mathbf{a})) \times \\
& \times \sum_{\mathbf{l}^{\prime}-\mathbf{a} \in[n,-\mathbf{a}], s^{\prime}} \varphi_{\mathbf{l}^{\prime}-\mathbf{a}, s^{\prime}}(\mathbf{r}) Q_{1 / 2}[n,-\mathbf{a}]_{\mathbf{l}^{\prime}-\mathbf{a}, s^{\prime} ; \mathbf{1}-\mathbf{a}, s},
\end{aligned}
$$

т.е.

$$
\psi_{\mathbf{k} s}^{[n]}(\mathbf{r}+\mathbf{a})=\exp (i \mathbf{k a}) \psi_{\mathbf{k} s}^{[n,-\mathbf{a}]}(\mathbf{r})
$$

и утверждение (10) доказано. 


\section{Список литературы}

[1] Займан Дж. Вычисление блоховских функций. М.: Мир, 1973.

[2] Troitskaya E. P. // Solid State Commun. 1984. V. 52. № 3. P. 321-323.

[3] Zarochentsev E. V., Tolpygo K. B., Troitskaya E. P. // Phys. Stat. Sol. (b). 1985. V. 127. № 2. P. $175-185$.

[4] Зароченцев Е.В., Троицкая Е. П. // ФТТ. 1985. Т. 27. №8. С. 2474-2478.

[5] Зароченцев Е. В., Троицкая Е. П., Файн Е.Я. // ФТТ. 1979. Т. 21. №2. С. 438-443.

[6] Lövdin P. O. Theoretical investigation into some properties of ionic crystals. Thesis. Uppsala, 1948.

[7] Троицкая Е. П., Еремейченкова Ю. В., Зароченцев Е. В. // ФТВД. Т. 4. № 3-4. С. 1-31.

[8] Abarenkov I. V., Antonova I. M. // Phys. Stat. Sol. 1970. V. 38. № 2. P. 783-797.

[9] Абаренков И. В., Антонова И. М. // ФТТ. 1978. Т. 20. № 2. С. 565-569.

[10] Абаренков И. В., Антонова И. М., Барьяхтар В.Г., Булатов В. Л., Зароченцев Е. В. Методы вычислительной техники в теории твердого тела. Электронная структура идеальных и дефектных кристаллов. Киев: Наукова думка, 1991.

[11] Борн М., Хуан Кунь. Динамическая теория кристаллических решеток. М.: ИИЛ, 1958.

[12] Троицкая Е. П. Основное и возбужденное состояния криокристаллов и их физические свойства. Дис. д-ра физ.-мат. наук. Киев, 1988.

[13] Зароченцев Е. В., Троицкая Е. П. // ФТТ. 1988. Т. 30. №8. С. 2367-2371.

[14] Ланкастер П. Теория матриц. М.: Наука, 1978.

Донецкий физико-технический институт НАН Украины

Поступила в редакцию 10.V.1995 г.

\section{Yu. V. Eremeichenkova, E. V. Zarochentsev, E. P. Troitskaya \\ A NEW CLUSTER-EXPANSION APPROACH TO THE CALCULATION OF THE ENERGY STRUCTURE OF CRYSTALS}

We propose a new approach to the energy structure calculation for strongly compressed or imperfect crystals in the case where the overlapping between localized orbitals (LO) of atoms with different space positions is large. This approach is based on the exact (in the sense of Lövdin) ortogonalization of LO for all atoms in crystal and on the cluster expansion (CE). In all $n$-th orders of CE we take into account overlapping integrals (OI) of LO for atoms, which belong to the same $n$-particle cluster. For every $n$ such clusters comprise a whole crystal. The CE converges rapidly as a power law in OI for $n \rightarrow \infty$. The two-particle cluster expansion is discussed in details. 